\title{
Dynamic iteration stopping algorithm for non-binary LDPC-coded high-order PRCPM in the Rayleigh fading channel
}

\author{
Rui Xue, Yanbo Sun* and Qiang Wei
}

\begin{abstract}
This paper mainly studies the association between non-binary low-density parity-check codes and high-order partial response continuous phase modulation, which prevents information loss in the mutual conversion of bit and symbol probabilities. Although the iterative detection and decoding technique applied in this system can obtain good performance/complexity tradeoff, the iterative process still encounters the problems of positive feedback and relatively large decoding delay, similar to other iterative coded modulation systems. The inhibitory effects of different extrinsic information exchange methods on positive feedback under different signal-to-noise ratio (SNR) conditions are investigated in this work to address this issue. Two dynamic iterative stopping algorithms, namely, cross entropy and hard decision aided combined with weighted extrinsic information exchange for cases with medium and high SNRs, are then proposed. Extrinsic information exchange between the demodulator and the decoder is conducted in the two algorithms through weighted processing. Iterative detection is subsequently performed based on two stopping criteria of dynamic iterative decoding. Theoretic analysis and simulation results for the Rayleigh fading channel show that the combination of weighted extrinsic information exchange and the two dynamic iterative stopping algorithms effectively resists positive feedback and improves the convergence of iterative detection and bit error rate performance. Such a combination also reduces the average iteration number to improve the real-time performance of iterative detection and decoding.
\end{abstract}

Keywords: Non-binary LDPC code, CPM, Convergence, Fading channel, Extrinsic exchange

\section{Introduction}

The two important design criteria in a wireless communication system include power and bandwidth efficiency. Continuous phase modulation (CPM) is a general class of constant envelope modulation that achieves high spectral efficiency with low spectral sidelobes by requiring a smooth phase transition between adjacent symbols [1]. These characteristics make CPM an ideal choice, with applications in different stringent communication systems employing nonlinear power amplifiers, such as satellite communication [2, 3], satellite mesh networks $[4,5]$ and satellite navigation [6, 7]. Rimoldi [8] presented a CPM modulator that can be decomposed into a cascade of a time-invariant convolutional encoder

*Correspondence: sunyanbo12345@126.com

College of Information and Communication Engineering, Harbin Engineering University, 150001 Harbin, China (continuous phase encoder, $\mathrm{CPE}$ ) operating on a ring of integers and of a time-invariant memoryless modulator (MM). In order to obtain further improvement in power efficiency, a forward error correcting (FEC) code is employed as an outer code to combine with CPM. This combination is the so-called serially concatenated CPM (SCCPM). A lot of work on the outer code has been recently carried out. Among the most attractive options emerged till now, a prominent role is played by convolutional codes $(\mathrm{CC})$, short binary low-density parity-check (LDPC) codes, parallel turbo codes (PTCs) and extended Bose-Chaudhuri-Hocquenghem (eBCH) code with soft-decision decoding [9].

Several binary FEC codes, such as turbo [10] and LDPC codes $[11,12]$ combined with CPM, can improve power efficiency and attain high error correcting capacity. However, the association between high-order CPM and binary codes suffers from significant information loss during the

\section{Springer}

(C) 2016 Xue et al. Open Access This article is distributed under the terms of the Creative Commons Attribution 4.0 International License (http://creativecommons.org/licenses/by/4.0/), which permits unrestricted use, distribution, and reproduction in any medium, provided you give appropriate credit to the original author(s) and the source, provide a link to the Creative Commons license, and indicate if changes were made. 
conversion from symbol probabilities to bit probabilities and its inversion in the process of transferring extrinsic information [13]. In addition, the convergence threshold of binary LDPC-coded CPM is higher than the Shannon limit. In view of these problems in binary-coded CPM, $q$ ary $(q>2)$ LDPC codes are introduced as the outer code scheme in this paper.

NB-LDPC codes outperform their binary counterparts in a block fading channel model, under which the channel periodically varies within a codeword [14]. Comparison with binary LDPC codes, PTCs and eBCH codes over jamming channels disturbed by pulsed jamming, continuous wave jamming or pseudo-noise jamming shows that NB-LDPC codes are the best solution to long code lengths [15]. Applying NB-LDPC codes allows for the design of efficient transmission schemes with high spectral efficiency [16]. Moreover, NB-LDPC-coded modulation exhibits lower receiver latency than binary LDPC-coded modulation, especially when complex equalization schemes are employed [17]. In our previous work [18], it has been shown that the combination of LDPC codes over GF $(q)$ and high-order PRCPM in additive white Gaussian noise (AWGN) channel achieves better tradeoff between power efficiency and bandwidth efficiency than binary LDPC-coded CPM.

Reference [19] reported that CPM is a promising solution for future satellite communication systems in the Ka band because it yields a constant envelope signal that enables the nonlinear power amplifier to operate near saturation. From the power efficiency point of view, a serially coded CPM can provide high power gain when the iterative decoding process is performed. The complete analysis in [2] has proven that SCCPM is a valid alternative scheme for the uplink of satellite communications regardless of the presence of broadband and narrowband transmissions. In view of the information loss in the combination of high-order CPM and binary codes, the NB-LDPC-coded high-order PRCPM scheme is considered a potential alternative for the uplink of satellite communications. Unfortunately, the scheme with iterative detection and decoding technique exhibits positive feedback and relatively large decoding delay during iterative detection, similar to other iterative coded modulation systems. To address these problems, two dynamic iterative stopping algorithms, namely, cross entropy (CE) and hard decision aided (HDA) based on weighted extrinsic information exchange, are proposed in this paper for cases with medium and high SNRs.

The rest of the paper is organized as follows. Section 2 introduces the system of the $q$-ary LDPC-coded PRCPM and the modified maximum a posteriori (MAP) algorithm for CPM in the Rayleigh fading channel. Section 3 investigates the convergence speed in the iteration and the performance of different extrinsic information exchange methods at various SNRs. Section 4 studies the technique of weighed extrinsic information exchange and its parameter setting. Section 5 expounds the CE and HDA dynamic iteration stopping algorithms. Section 6 discusses the simulations. Finally, we conclude the paper in Section 7.

\section{System description}

\subsection{System of NB-LDPC-coded PRCPM}

The $M$-ary CPM signal in [20] is described as follows:

$s(t, \chi)=\sqrt{2 E_{s} / T} \cos \left(2 \pi f_{0} t+\psi(t, \chi)+\psi_{0}\right), \quad t \geq 0,(1)$

where $f_{0}, \psi_{0}$ and $\chi$ denote the carrier frequency, initial phase shift and sequence of the $M$-ary symbol $\left(\chi_{i} \in\right.$ $\{ \pm 1, \pm 3, \ldots \pm(M-1)\})$, respectively. $T$ and $E_{s}$ denote the symbol period and energy, respectively. $\psi(t, \chi)$ denotes the information carrying phase defined as

$$
\psi(t, \chi)=2 \pi h \sum_{i=0}^{\infty} \chi_{i} q(t-i T)
$$

where $h=k / p$ ( $k$ and $p$ are relatively prime positive integers) is the modulation index; $q(t)$ is the integral of a positive normalized frequency pulse $g(t)$; and $g(t)$ is nonzero for $L$ symbol periods, with a full response for $L=1$ and a partial response for $L>1$. The physical tilted phase is provided by [8] as

$$
\begin{aligned}
\phi(\tau+n T, \mathbf{U})= & R_{2 \pi}\left[2 \pi h R_{p}\left[\sum_{i=0}^{n-L} U_{i}\right]+4 \pi h \sum_{i=0}^{L-1} U_{n-i} q\right. \\
& \times(\tau+i T)+W(\tau)], 0 \leq \tau<T
\end{aligned}
$$

where $U_{i}=\left(\chi_{i}+(M-1)\right) / 2, R_{x}[\cdot]$ is a modulo- $x$ function and $W(\tau)$ is a data-independent function expressed as follows:

$$
\begin{aligned}
W(\tau)= & \pi h(M-1) \tau / T-2 \pi h(M-1) \sum_{i=0}^{L-1} q(\tau+i T) \\
& +\pi h(L-1)(M-1), 0 \leq \tau<T .
\end{aligned}
$$

The following transmitted signal is expressed when Eqs. (1) to (4) are combined.

$$
s(t, \mathbf{U})=\sqrt{2 E_{s} / T} \cos \left(2 \pi f_{1} t+\phi(t, \mathbf{U})+\psi_{0}\right),
$$

where $f_{1}=f_{0}-h(M-1) / 2 T$. The transmitted signal is completely specified by the current symbol $U_{n}$, the $L-1$ previous data symbols $\left[U_{n-1}, \ldots, U_{n-L+1}\right]$, and the accumulated value of $V_{n}=R_{p}\left(\sum_{i=0}^{n-L} U_{i}\right)$. Therefore, CPM is decomposed as a CPE followed by an MM. 
CPE is regarded as a convolutional code with a code rate of " 1 " because of its memorial and recursive characteristics. The model of $q$-ary LDPC-coded $M$-ary CPM over a Rayleigh fading channel with iterative decoding is established when the CPE is viewed as an inner code serially concatenated with the $q$-ary LDPC encoder (Fig. 1).

For a particular code rate $R$ and codeword length $N$, a sparse parity check matrix $\mathbf{H}=\left[h_{i, j}\right]_{P \times N}(P=N(1-R))$ with $h_{i, j} \in \mathrm{GF}\left(q=2^{b}\right)$ ( $b$ is a positive integer) is needed to construct. In the transmitter, input information symbol vector $\mathbf{U}^{o}=\left(U_{0}^{o}, U_{1}^{o}, \cdots, U_{K-1}^{o}\right)$ of size $K=N-P$ is encoded by the $q$-ary LDPC encoder into codeword $\mathbf{C}^{o}=\left(C_{0}, C_{1}, \cdots, C_{N-1}\right)$. Codeword $\mathbf{C}^{o}$ is then sent to the interleaver, whose output is then mapped to $\mathbf{U}^{i}$ (e.g. Gray mapping) if the finite field size of the NB-LDPC encoder is not equal to the alphabet size of CPM. CPE subsequently produces inner code symbol vector $\mathbf{C}^{i}$, which goes through MM to form modulated signal vector $s(t, \mathbf{U})$. In this paper, we always assume that $q$ is equal to $M$, and the symbol mapping module is unnecessary. For convenience, we use the notation " $L R C$ " for a raised cosine pulse of length $L$ symbol intervals. Complex signal vector $s(t, \mathbf{U})$ is then finally transmitted over a Rayleigh fading channel.

The iterative receiver mainly consists of two soft-input soft-output (SISO) decoders for the inner CPM and an outer $q$-ary LDPC encoder. Demodulation and decoding are performed by iteration (called "outer iteration") between CPM-SISO and LDPC-SISO. The Log-MAP algorithm is employed by the CPM-SISO subsystem. A log-domain belief propagation iterative decoding algorithm (called "inner iteration") combined with fast Fourier transform simplified as Log-FFT-BP [21] is performed in the LDPC-SISO subsystem. LDPC-SISO in the investigated system is run with five times inner iterations per outer iteration. The SISO algorithm is a nice generalization of the BCJR algorithm, which takes a priori probabilities of both information and code symbols and computes extrinsic a posterior probabilities (APPs) of both information and code symbols [22], as shown in
Fig. 1. The decision device finally selects the information symbol with the maximum APP in the last iteration.

\subsection{Modified MAP algorithm for CPM in the Rayleigh fading channel}

CPE can be represented by a trellis if modulation index $h(h=k / p)$ is a rational number and when $k$ and $p$ are relatively prime positive integers. The SISO algorithm can be applied to the trellis if only the proper transition probability distribution $p\left(\underline{r}_{n} \mid \underline{c}_{n}\right)$ is used in the branch metric $\gamma_{n}=p\left(\underline{r}_{n} \mid \underline{c}_{n}=C\right) \cdot \operatorname{Pr}\left(U_{n}=U\right)$ [22]. Accordingly, $\underline{r}_{n}$ is a sufficient statistic based on channel observations during symbol interval $n, U \in \mathbf{U}^{i}, C \in \mathbf{C}^{i}$. The transition probability distribution $p\left(\underline{r}_{n} \mid \underline{c}_{n}\right)$ is a joint probability density function (PDF) conditioned on the CPE code symbol $\underline{c}_{n}$. A bank of $p M^{L}$ complex filters is matched to the CPM signals in each symbol interval and sampled once every symbol interval to produce a sufficient statistic.

We let the output of these filters sampled at time $t=$ $(n+1) T$ constitute sufficient statistic (vector) $\underline{r}_{n}$ with mean vector $\underline{m}_{n}$ and covariance matrix $\underline{\Lambda}$. Given that the components of $\underline{r}_{n}$ are Gaussian and partially correlated in all hypotheses, their joint PDF conditioned on the transmission of $\underline{c}_{n}$ is described as [23]

$$
p\left(\underline{r}_{n} \mid \underline{c}_{n}\right) \propto \exp \left\{-\left(\underline{r}_{n}-\underline{m}_{n}\right)^{H} \Lambda^{-1}\left(\underline{r}_{n}-\underline{m}_{n}\right)\right\},
$$

where $(\cdot)^{H}$ is the conjugate transposition operator.

A Rayleigh fading channel is regarded as a memoryless channel if interleaving is sufficient. We adjust the calculation methods of transition probability distribution over a slow Rayleigh fading channel as follows. If channel state information (CSI) $\underline{a}_{n}$ is known for the receiver, $p\left(\underline{r}_{n} \mid \underline{c}_{n}\right)$ is modified as

$$
p\left(\underline{r}_{n} \mid \underline{c}_{n}\right) \propto \exp \left\{-\left(\underline{r}_{n}-\underline{a}_{n} \underline{m}_{n}\right)^{H} \Lambda\left(\underline{a}_{n}\right)^{-1}\left(\underline{r}_{n}-\underline{a}_{n} \underline{m}_{n}\right)\right\}
$$

where $\underline{a}_{n} \underline{m}_{n}$ is the mean vector of the matched filter output. The mean of the Rayleigh fading amplitude is used,

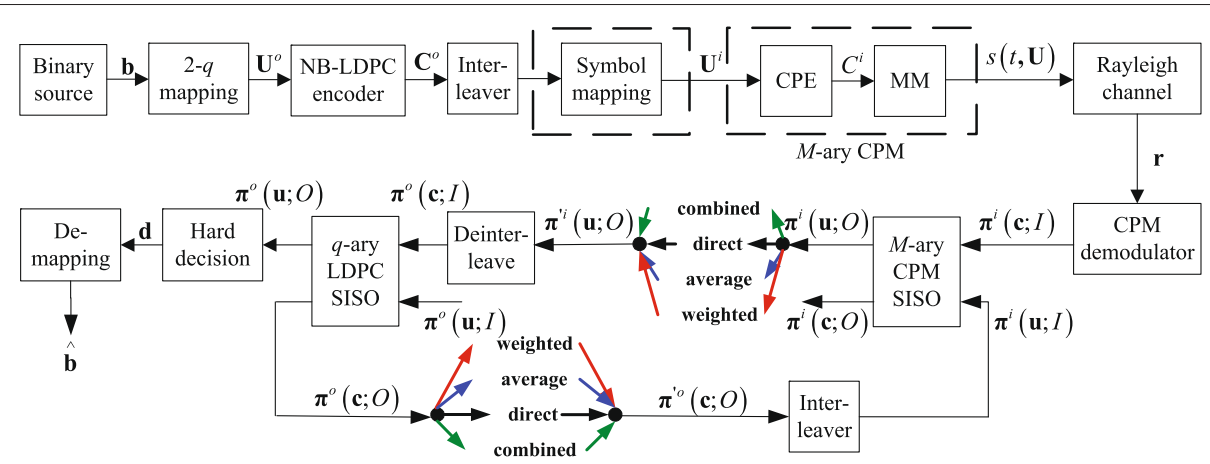

Fig. 1 System model of $q$-ary LDPC-coded M-ary PRCPM 
and $\underline{r}_{n}$ is assumed to be a joint Gaussian distribution if the CSI is unknown for the receiver. $p\left(\underline{r}_{n} \mid \underline{c}_{n}\right)$ is then computed as follows:

$$
p\left(\underline{r}_{n} \mid \underline{c}_{n}\right) \propto \exp \left\{-\left(\underline{r}_{n}-E_{A}(a) \underline{m}_{n}\right)^{H} \Lambda\left(\underline{a}_{n}\right)^{-1}\left(\underline{r}_{n}-E_{A}(a) \underline{m}_{n}\right)\right\}
$$

where $E_{A}(a)$ is the mean of the Rayleigh fading amplitude.

\section{Analysis of the iterative detection process}

\subsection{Convergence of iteration}

Although maximum likelihood sequence detection over all the concatenated elements is the optimum detector, the process is often too complex to realize. Turbo decoding provides a sub-optimum but often realizable approximation [24]. The iterative detection of a non-binary LDPCcoded high-order CPM with interleaving is examined in this study. The channel encoding and CPM modulation operations for coded data over narrowband channels are viewed as a serial concatenation of two finite-state machines separated by an interleaver. Joint demodulation and decoding are iteratively performed using the extrinsic information exchange between a CPM-SISO demodulator and a SISO channel decoder [25].

The abovementioned analysis shows that the iterative detection mechanism for demodulation and decoding is established on the basis of interchanging and transferring extrinsic information. The extrinsic information in each iterative process is the extra information that the individual SISO decoder obtains in the iterative process. This information has nothing to do with the system information and priori information. The extrinsic information is delivered to the other sub-decoder as a priori information through interleaving (or deinterleaving). Decoding performance is improved by exchanging extrinsic information. Therefore, extrinsic information is a key factor in iterative decoding.

During the process of research, we have observed that the iterative detection in $q$-ary LDPC-CPM has the phenomenon of positive feedback; in other words, the bit error rate (BER) performance degrades with increasing iterations. Figure 2 reports the convergence speed of the 8-ary LDPC-coded PRCPM in a slow flat Rayleigh fading channel. The information frame length in this simulation is 384 bits, and the code rate of the 8 -ary LDPC code with variable node degree distribution $\lambda(x)=0.38354 x+$ $0.04237 x^{2}+0.57409 x^{3}$ is $1 / 2$. The random interleaver and Gray mapping are employed. The inner iteration number is set to 5 . No CSI is available, and $E_{A}(a)=0.8862$. An excellent implementation scheme denoted by $8 M 2 \mathrm{RC}$ ( $h=0.5$ ) is considered for the PRCPM modulator. This scheme provides a good tradeoff between power efficiency and bandwidth efficiency [11]. 8M2RC indicates that alphabet size $M$ is equal to 8 , and the frequency pulse is a raised cosine (RC) with length $L=2$. Figure 2a clearly shows that the positive feedback phenomenon is obvious in the investigated system. The BER curves are prone to increase with a certain number of iterations for $E_{b} / N_{0}$ of 1.4 and $2 \mathrm{~dB}$. Moreover, the positive feedback is no longer outstanding after approximately 10 iterations and the trend then begins to fluctuate at a pool BER level. However, BER performance is improved by the increase in the iteration number (Fig. $2 b$ ) when $E_{b} / N_{0}$ is varied from 3 to $4 \mathrm{~dB}$. Analysis shows that the positive feedback phenomena becomes increasingly serious in the region with low SNR.

The phenomenon existing in $q$-ary LDPC-CPM can be explained by the theory of discrete-time dynamical system defined on a continuous set [26]. Similar to SCCPM systems, the phase trajectories of $q$-ary LDPC-CPM mainly fall into one of the following three modes: (1) convergence to an unequivocal fixed point; (2) convergence to an indecisive fixed point; and (3) convergence to a fixed set. The majority of frames belong to mode 1 or 2 . Mode 3 mostly occurs in the waterfall region. The interleaving for the short frame $q$-ary LDPC-CPM system is insufficient. Furthermore, the possibility of burst error remains high, which makes BER oscillation more serious than in the long frame ones. Positive feedback in the BER of the system arises when the number of oscillation frames is sufficiently large.

\subsection{Exchange methods of extrinsic information}

Iterative detection is a key technology to improve BER performance and reduce realization complexity in turbolike receivers. Iteration between the demodulator and the decoder is established by transferring extrinsic information. Current research reveals the following three main exchange methods of extrinsic information (i.e. direct exchange, average exchange and weighted exchange).

(1) Direct exchange is an original method, which is also called simple exchange. The extrinsic information provided by the previous SISO is interleaved or de-interleaved and then directly transmitted to the other SISO decoder without any processing as a prior information (Fig. 1). This method is expressed as follows:

$$
\begin{array}{r}
\pi^{\prime}\left(\mathbf{u}^{\mathbf{j}} ; O\right)=\pi\left(\mathbf{u}^{\mathbf{j}} ; O\right), \\
\pi^{\prime}\left(\mathbf{c}^{\mathbf{j}} ; O\right)=\pi\left(\mathbf{c}^{\mathbf{j}} ; O\right),
\end{array}
$$

where $\pi\left(\mathbf{u}^{\mathbf{j}} ; O\right)$ and $\pi\left(\mathbf{c}^{\mathbf{j}} ; O\right)$ denote the probability distributions of information and code symbols in the log-domain, respectively.

(2) Average exchange takes the mean of the extrinsic information exported by one SISO decoder in the 

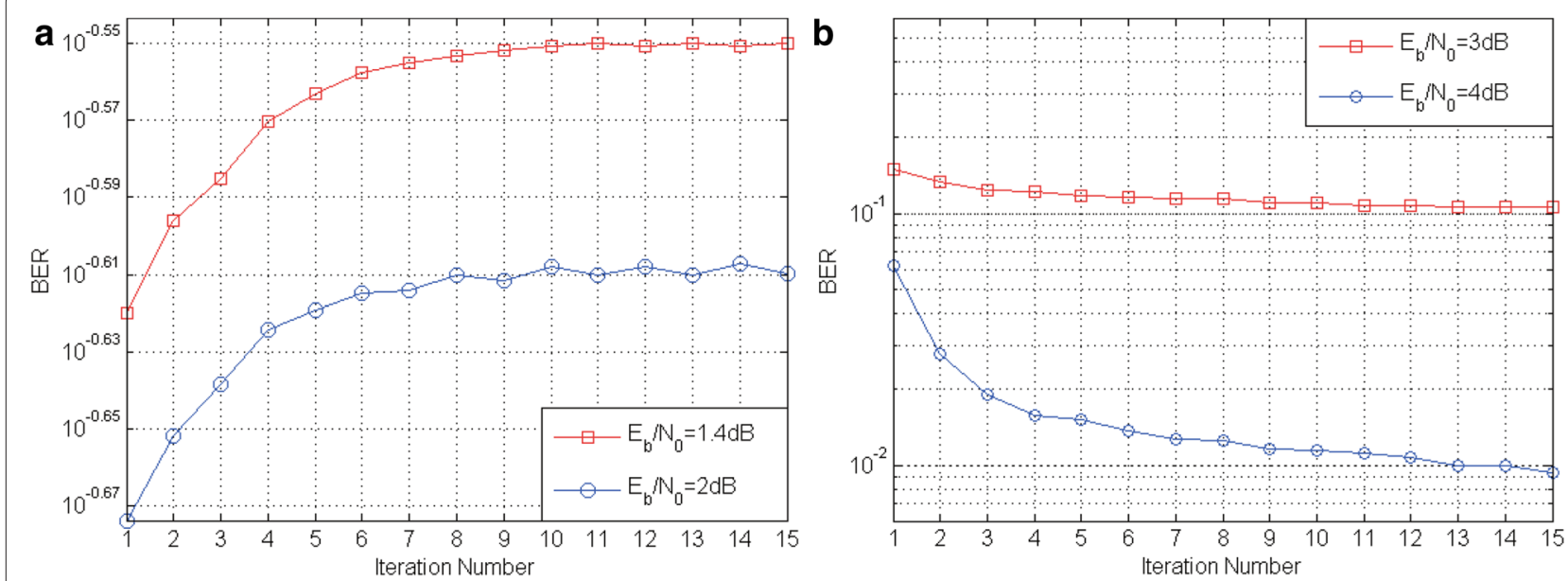

Fig. 2 Iterative convergence speed of the 8-ary LDPC-8M2RC over the Rayleigh fading channel for different SNRs. $\mathbf{a} \mathrm{E}_{\mathrm{b}} / \mathrm{N}_{0}=1.4$ and $2 \mathrm{~dB}$. b $\mathrm{E}_{\mathrm{b}} / \mathrm{N}_{0}=3$ and $4 \mathrm{~dB}$

previous iterations as a prior information in the current iteration. This method is expressed as follows:

$$
\begin{aligned}
& \pi^{\prime(l)}\left(\mathbf{u}^{\mathbf{j}} ; O\right)=\log \left(\frac{1}{l} \sum_{i=1}^{l} \exp \left(\pi^{(i)}\left(\mathbf{u}^{\mathbf{j}} ; O\right)\right)\right), \\
& \pi^{\prime(l)}\left(\mathbf{c}^{\mathbf{j}} ; O\right)=\log \left(\frac{1}{l} \sum_{i=1}^{l} \exp \left(\pi^{(i)}\left(\mathbf{c}^{\mathbf{j}} ; O\right)\right)\right),
\end{aligned}
$$

where $\pi^{(i)}\left(\mathbf{u}^{\mathbf{j}} ; O\right)$ and $\pi^{(i)}\left(\mathbf{c}^{\mathbf{j}} ; O\right)$ are the log-domain probability distributions of information and code symbols in the $i$ th iteration. $l$ denotes the present number of iterations.

(3) In weighted exchange, the extrinsic information exported by the former decoder is delivered to the weighting function instead of being directly transmitted to the next decoder. More details on the weighted exchange method are discussed in Section 4.

Both average and weighted methods inhibit the positive feedback phenomenon and effectively improve BER performance in convolutional coded CPM systems [27, 28]. Hence, in this study, we combine the average method with the weighted method (i.e. the mean of extrinsic information is derived by weighting before transmitting to the next decoder). A comparative study of different extrinsic information exchange methods in $q$-ary LDPC-CPM systems is one of the main contents of this paper.

Figure 3 shows a comparison of iterative convergence speed in an 8-ary coded 8M2RC system with the direct, average, weighted and combined methods when $\mathrm{E}_{\mathrm{b}} / \mathrm{N}_{0}$ is set to 0.4 and $4.4 \mathrm{~dB}$. All the other simulation parameters are similar to those in the settings in Fig. 2.
Figure 3a shows that the average and combined methods both inhibit positive feedback to some extent at $\mathrm{E}_{\mathrm{b}} / \mathrm{N}_{0}=0.4 \mathrm{~dB}$, avoid violent $\mathrm{BER}$ oscillation and maintain BER at a stable level with increasing iteration number. All the four methods effectively inhibit positive feedback, and BER continuously decreases with increasing iteration number (Fig. 3b). The weighted method exhibits the best iterative convergence and the lowest BER at the same iteration compared with other methods at $\mathrm{E}_{\mathrm{b}} / \mathrm{N}_{0}=4.4 \mathrm{~dB}$. For instance, the BER of $8.26 \times 10^{-4}$ is attainable for the weighted exchange method at the seventh iteration, whereas the BER for the direct method is only $2.91 \times 10^{-3}$. Notably, the BER performances of the average and combined methods are worse than that of the direct exchange method at high SNR. Figure 4 shows a comparison of BER in the four methods when the outer iteration number is uniformly fixed at 8 . All the other parameters are similar to those in the former. The BER performance order of different methods is expressed as follows when $\mathrm{E}_{\mathrm{b}} / \mathrm{N}_{0}$ is changed from 2.8 to $4.8 \mathrm{~dB}$ (Fig. 4): weighted method $>$ direct method $>$ combination method $>$ average method. Thus, weighted exchange is an effective method to improve the power efficiency of the investigated scheme.

\section{Weighted exchange method of extrinsic information}

The simulation results in our previous work [12] indicated that the weighted exchange of extrinsic information in binary LDPC-coded CPM over a Rayleigh fading channel effectively inhibits the positive feedback phenomenon and improves the BER performance under the condition of low SNR. Comparison with the direct exchange method shows that the weighted exchange method can attain a $0.2 \sim 0.3 \mathrm{~dB}$ gain at $\mathrm{BER}=10^{-3}$ with an information block 

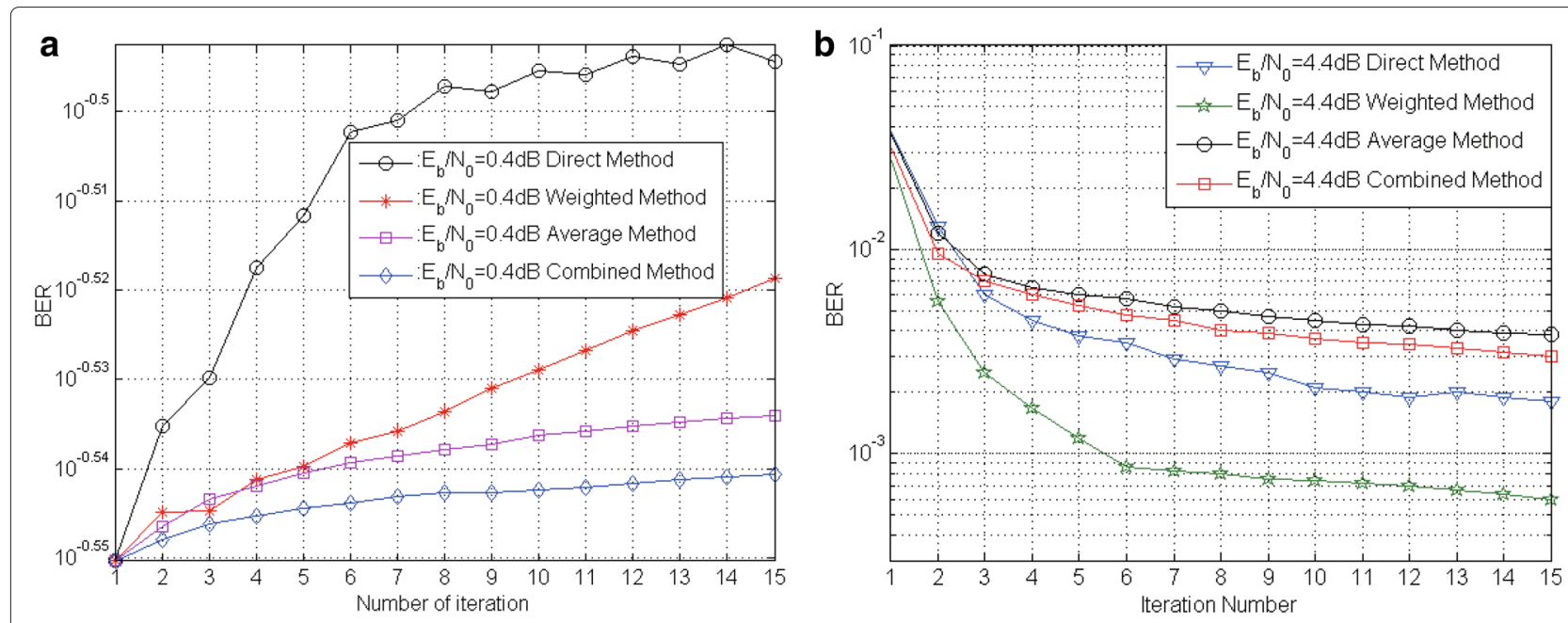

Fig. 3 Iterative convergence speed of the four exchange methods based on 8-ary LDPC-coded $8 M 2 R C$ over the Rayleigh fading channel. a $E_{b} / N_{0}=$ $0.4 \mathrm{~dB} \cdot \mathbf{b} \mathrm{E}_{\mathrm{b}} / \mathrm{N}_{0}=4.4 \mathrm{~dB}$

length of 384 bits and a modulation index of $1 / 2$ when the iteration number is varied from 5 to 15 . Inspired by the study of Xue et al. [12], we design a method based on weighted extrinsic information probability for $q$-ary LDPC-CPM systems.

The CPM and LDPC decoders compute the probability distributions of information and code symbols according to the additive SISO (A-SISO) based on the Log-MAP algorithm presented in [29] as follows:

$\pi_{k, j}\left(u^{j}, O\right)=\log \left[\sum_{u: U_{k}^{j}=w^{j}} \exp \left\{\pi_{\mathbf{k}}(\mathbf{u} ; O)+\sum_{i=1, i \neq j}^{k_{0}} \pi_{k, j}\left(u^{i} ; I\right)\right\}\right]$,

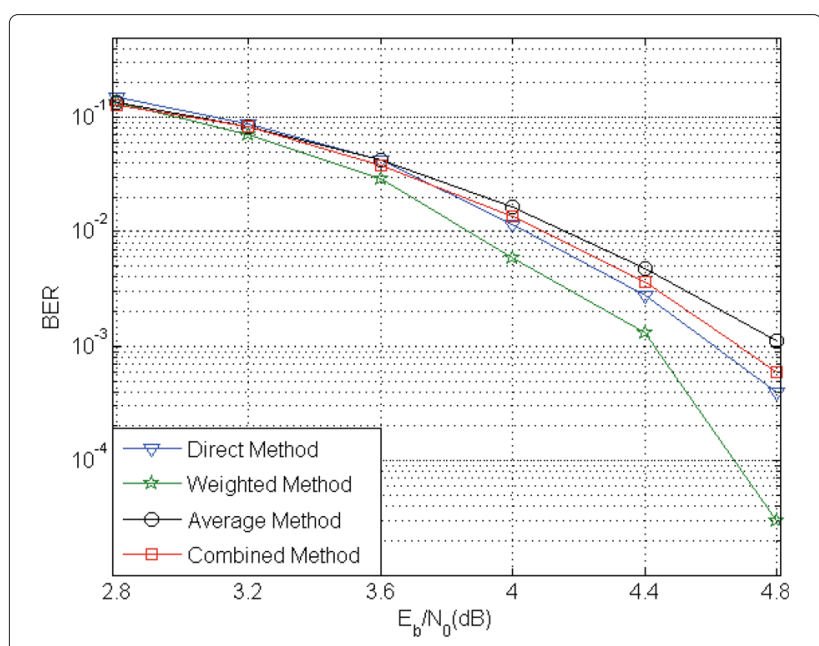

Fig. 4 BER performance of the four exchange methods $\pi_{k, j}\left(c^{j}, O\right)=\log \left[\sum_{c: C_{k}^{j}=c^{j}} \exp \left\{\pi_{\mathbf{k}}(\mathbf{c} ; O)+\sum_{i=1, i \neq j}^{n_{0}} \pi_{k, j}\left(c^{i} ; I\right)\right\}\right.$,

where $\pi_{\mathbf{k}}(\mathbf{u} ; O)$ and $\pi_{\mathbf{k}}(\mathbf{c} ; O)$ indicate the probabilities of information and code symbols at time $k$, respectively. $k_{0}$ and $n_{0}$ denote the length of a single information and the code symbol, respectively.

Figure 1 shows that the extrinsic information probabilities of the inner SISO output are sent to the weighted function module before being delivered to the outer SISO. Similarly, the extrinsic information probabilities of the outer SISO output are sent to the weighted function module before being delivered to the inner SISO.

$$
\begin{gathered}
\pi^{\prime}\left(\mathbf{u}^{\mathbf{j}} ; O\right)=\psi\left(\pi\left(\mathbf{u}^{\mathbf{j}} ; O\right)\right), \\
\pi^{\prime}\left(\mathbf{c}^{\mathbf{j}} ; O\right)=\psi\left(\pi\left(\mathbf{c}^{\mathbf{j}} ; O\right)\right),
\end{gathered}
$$

where $\psi(\cdot)$ denotes the weighted function of size $q \times$ $N$, and $*$ indicates point multiplication. $\psi(\cdot)$ is used as the function of the extrinsic information probabilities for convenience and to adaptively adjust the extrinsic information according to its size.

$$
\psi(\pi)=a \pi \cdot * \exp (-\beta|\pi|)
$$

where $\alpha$ and $\beta$ denote the weighted parameters decided by the interleaver length, SNR and other parameters through simulation experiments. The iterative decoding scheme based on the weighted extrinsic information method is shown in Fig. 5.

A comparative analysis of the computational complexity of the four methods is provided in Table 1 . The computational complexities of CPM-SISO and LDPC-SISO in the receiver are also displayed in the same table, where $w_{c}$ is 


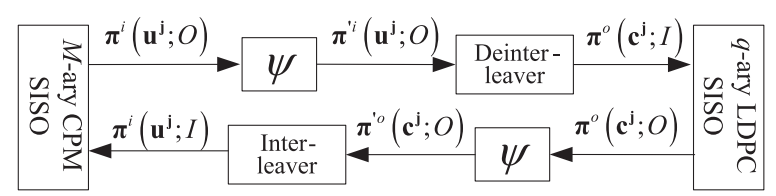

Fig. 5 Principle diagram of iterative detection based on the weighted method

the uniform column weight of sparse parity check matrix $\mathbf{H}$ and $n_{\text {inner }}$ denotes the inner iterations for LDPC-SISO. The weighted method obviously has a moderate computational complexity that is comparable to that of the average method and much lower than that of the combined method. Compared with that of the direct method, the computational complexity of the weighted method only increases by $6 M$ in multiplications and $2 M$ in log\&exp operations and is far less than that of CPM-SISO. Therefore, the entire computational complexity for coded CPM schemes mainly depends on the CPM demodulator. The computational complexity also linearly increases with the iteration number. A preferable tradeoff between computational complexity and BER performance should be considered. Two dynamic iterative stopping algorithms based on weighted extrinsic information exchange are proposed in this study to address this problem. More details are shown in Section 5.

From Eq. (17), it results that the weighted parameter is the key factor that affects performance. Excellent performance has been obtained with values of $\alpha \in[0.6,0.9]$ and $\beta \in[0.001,0.01]$ in similar coded CPM systems $[10,12]$. However, the specific $\alpha$ and $\beta$ values in the system we investigate need to be determined using simulation experiments. Figure 6 reports the BER performance with various weighted parameter combinations of $\alpha$ and $\beta$. The length of the information frame in the simulation is 768 bits. The code rate of the 8 -ary LDPC code with variable node degree distribution $\lambda(x)=0.1290 x+0.4839 x^{2}+$ $0.3871 x^{5}$ is $2 / 3$. The other simulation parameters are the same as before. Figure 6 shows that the BER curves with $\alpha \in[0.6,0.9]$ and $\beta \in[0.001,0.01]$ almost coincide when $\mathrm{E}_{\mathrm{b}} / \mathrm{N}_{0}$ is in the $3.2 \sim 4.4 \mathrm{~dB}$ region. The BER performance of the weighted method with different parameters is much better than that of the direct method. The combination of $\alpha=0.7$ and $\beta=0.01$ is selected because of the fact that the smallest BER of $8.68 \times 10^{-6}$ is achievable at $E_{b} / N_{0}$ of $4.4 \mathrm{~dB}$.

\section{Iterative stopping algorithm}

The number of outer iterations in the iterative detection process of $q$-ary LDPC-CPM systems is usually set to a fixed positive integer. However, not all received sequences have optimal decoding results at the same number of iterations. For several specific sequences, error-free decoding can be achieved by a few iterations. Continuous iteration would increase computational complexity and iterative decoding delay. A dynamic iteration would possibly improve detection efficiency and reduce decoding delay if some iterative stopping criteria are introduced into the detection process. In this study, cross entropy (CE) [30] and hard decision-aided (HDA) [31] stopping criteria are incorporated into the iterative process. Two dynamic iteration stopping algorithms based on weighted extrinsic information exchange are then developed. The application of the two criteria in $q$-ary LDPC-CPM systems' signal detection is subsequently deduced.

\subsection{CE stopping criterion}

The CE stopping criterion, which was first proposed in turbo code by J. Hagenauer, has been widely applied in iterative decoding. S. Zhang successfully introduced the criterion into bit-interleaved coded modulation with iterative decoding [32]. A variety of improved stopping criteria based on CE (e.g. sign change ratio [33] and HAD) have been introduced successively since then.

We utilize the probability matrix of information symbols $\left[P(u ; O)_{m, k}\right]_{M \times K}$ exported by the $M$-ary LDPC-SISO module in two adjacent iterations to compute the mean of $\mathrm{CE}, T(i)$, directly and to avoid the mutual conversion between likelihood ratio and probability.

$$
T(i)=\frac{1}{K} \sum_{k=0}^{K-1} \sum_{m=0}^{M-1} P^{i}(u ; O)_{m, k} \log \frac{P^{i}(u ; O)_{m, k}}{P^{i-1}(u ; O)_{m, k}}, i \geq 2,
$$

where $M$ is the alphabet size of CPM, $K$ is the length of the information symbol vector, and $P^{i}(u ; O)_{m, k}$ denotes the

Table 1 Computational complexity of q-ary LDPC-coded PRCPM using various extrinsic information exchange methods

\begin{tabular}{llll}
\hline Per symbol per iteration & Additions & Multiplications & Log\&exp operations \\
\hline Direct method & 0 & 0 & 0 \\
Weighted method & 0 & $6 M$ & $2 M$ \\
Average method & $2 M$ & $2 M$ & $6 M$ \\
Combined method & $2 M$ & $8 M$ & $8 M$ \\
CPM-SISO & $3 p M^{L-1}(3 M+1)$ & $3 p M^{L-1}$ & $3 p M^{L-1}(M+1)$ \\
LDPC-SISO & 0 & $\left(2 q \log _{2} q+4 q\right) w_{c} n_{\text {inner }}$ & 0 \\
\hline
\end{tabular}



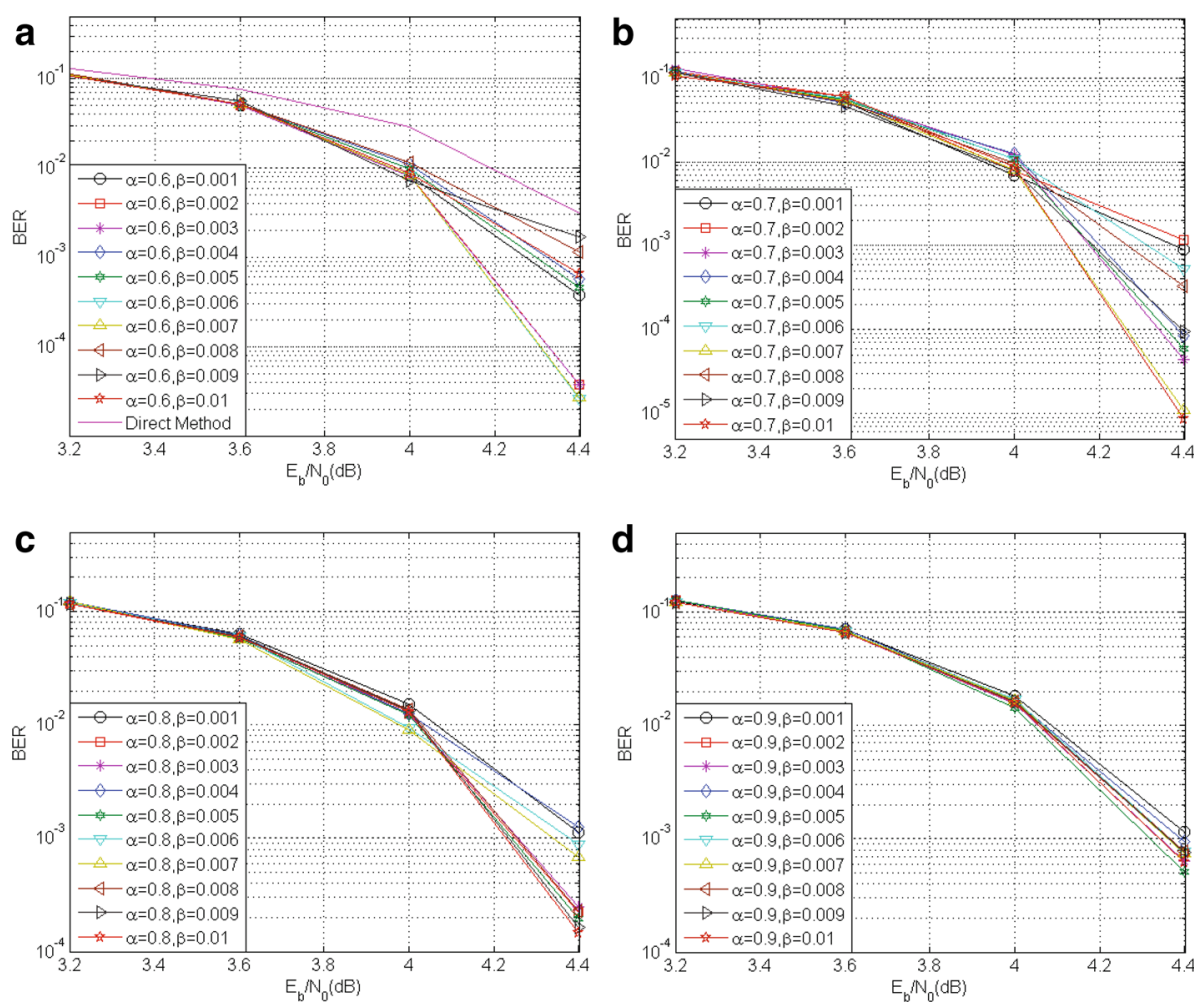

Fig. $6 \mathrm{BER}$ versus $\mathrm{E}_{\mathrm{b}} / \mathrm{N}_{0}$ as parameterized by the weighted parameters. $\mathbf{a} \alpha=0.6, \mathbf{b} \alpha=0.7, \mathbf{c} \alpha=0.8$ and $\mathbf{d} \alpha=0.9$

probability of the event that the $k$ th element in the information symbol vector is equal to $(m-1)$. The threshold of the CE stopping criterion is expressed as follows:

$$
T(i) \leq w T(1)
$$

where $w$ is the adjusting parameter. Table 2 shows the effects of adjusting parameters on system performance with the $\mathrm{CE}$ stopping criterion at $\mathrm{E}_{\mathrm{b}} / \mathrm{N}_{0}=4 \mathrm{~dB}$. The combination of $\alpha=0.7$ and $\beta=0.01$ is selected. The other simulation parameters are similar to those in Fig. 6. Table 2 shows that the average iteration number gradually increases with the decrease in $w$. Meanwhile, BER is continuously reduced. The improvement in BER is no longer prominent once $w$ approaches a fairly small order of magnitude. Therefore, the value of $w$ should be determined by a specific requirement in different systems.

\subsection{HDA stopping criterion}

In each iteration, the information bit sequence $\left[\hat{b}_{k}\right]$, namely, the output of the demapper, whose input is the information symbol hard decision sequence exported by the $q$-ary LDPC-SISO module can predict convergence during the iterative decoding process. The iteration process is considered convergent if the sign of the information bit sequence does not change in two adjacent iterations.

$$
\left[\hat{b}_{k}\right]^{i-1} \oplus\left[\hat{b}_{k}\right]^{i}=0
$$

The HDA stopping criterion is introduced into the $q$-ary LDPC-CPM systems. Furthermore, the iterative stopping condition is simplified by counting the number $D(i)$ of the event where the sign of the information sequence is different in two adjacent iterations. In other words, the iteration is stopped if $D(i) \leq Q \times K \times \log _{2} M$; otherwise, the iteration continues. Accordingly, $Q$ is defined as a constant with a general range of $[0.001,0.01]$, and $K$ is the length of the information symbol block.

Table 2 BER performance of different adjusting parameters

\begin{tabular}{llllll}
\hline Adjusting parameters & $w=10^{-1}$ & $w=10^{-2}$ & $w=10^{-3}$ & $w=10^{-4}$ & $w=10^{-5}$ \\
\hline Average iteration number & 5.150 & 5.435 & 5.580 & 5.785 & 5.805 \\
BER & 0.0073 & 0.0062 & 0.0057 & 0.0052 & 0.0051 \\
\hline
\end{tabular}




\section{Simulation results}

The validity of the dynamic iterative stopping algorithms combined with weighted extrinsic information exchange is then tested. Monte Carlo simulations based on MATLAB software are performed to evaluate the performance of the proposed systems in the Rayleigh fading channel (i.e. slow flat Rayleigh fading channel, $E_{A}(a)=0.8862$, and no CSI is available). The other simulation parameters are similar to those in Fig. 6. More details on the parameter settings are discussed in Section 4.

Figure 7 shows the BER performance of the CE and HDA iterative stopping algorithms combined with the direct and weighted methods in the 8-ary LDPC-8M2RC system. The outer iteration number is set to 16 . Accordingly, $w=10^{-4}$ and $Q=0.01$ are separately employed in the CE and HDA iterative stopping algorithms. The BER performance of the CE and HDA iterative stopping algorithms based on the direct method (abbreviated as CEdirect and HDA-direct, respectively) exhibits a negligible degradation with respect to the direct method alone. Similarly, the CE and HDA iterative stopping algorithms based on the weighted method (abbreviated as CE-weighted and HDA-weighted, respectively) exhibit minor BER performance degradation with respect to the weighted method alone. Notably, the BER of the CE-direct and HDA-direct algorithms gradually declines when $\mathrm{E}_{\mathrm{b}} / \mathrm{N}_{0}$ is more than $4.4 \mathrm{~dB}$ and begins to step into the error floor region. Compared with the direct method, the weighted method achieves about $0.2 \mathrm{~dB}$ gain at $\mathrm{BER}=10^{-3}$. The BER of the CE-weighted and HDA-weighted algorithms continuously decreases when $\mathrm{E}_{\mathrm{b}} / \mathrm{N}_{0}$ exceeds $4.4 \mathrm{~dB}$ and effectively avoids entering the error floor region. The reason is that the weighted method prevents the positive feedback phenomenon from occurring. Moreover, the CE and

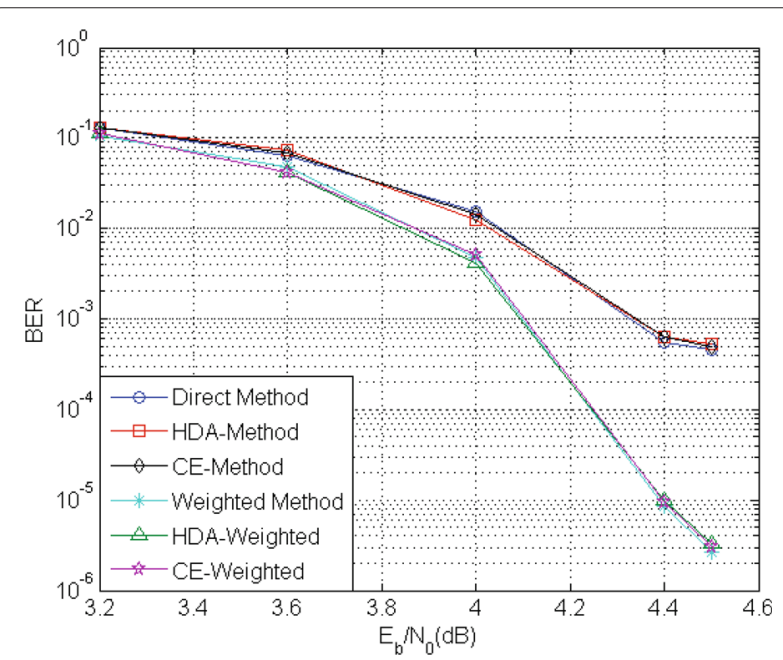

Fig. 7 BER performance of the CE-weighted and HDA-weighted algorithms
HDA iterative stopping algorithms stop iterations in time before going into positive feedback. The weighted method improves the iterative convergence to reach a low error rate as SNR increases.

Figure 8 shows the average iteration number of the $\mathrm{CE}$-weighted and HDA-weighted algorithms. The average iteration number of the two proposed algorithms gradually decreases with the increase in SNR. Accordingly, 4.175 and 3.665 iteration times are separately attainable for the CE-direct and HDA-direct algorithms at $\mathrm{E}_{\mathrm{b}} / \mathrm{N}_{0}$ of $4.4 \mathrm{~dB}$. Meanwhile, 3.88 and 3.38 iteration times are respectively available for the CE-weighted and HDAweighted algorithms at the same SNR. Analysis of Figs. 8 and 9 shows that the CE-weighted and HDA-weighted algorithms reduce the average iterative number and computational complexity. These algorithms also significantly improve the performance of BER and real-time in iterative detection compared with the direct method alone. Therefore, the combination of weighted extrinsic information exchange and the two iterative stopping algorithms can offer double insurance in enhancing reliability and reducing the decoding delay.

For a fair comparison, several alternative schemes provided by literature are selected from several similar coded CPM systems to investigate the BER performance of 8-ary LDPC-coded $8 M 2 R C$. The turbo-CPM scheme is discussed in [10], where a constituent encoder with the generator polynomial $(10,04,15)$ in octal representation is employed by turbo. Convolutional coded CPM (CCCPM) schemes are studied in [23] and [34], where the generator polynomials of CC are $(13,06,16)[23]$ and $(11$, 06, 16) [34], respectively. The binary LDPC-coded CPM (BLDPC-CPM) scheme is considered in [35] with the same variable node degree distribution $\lambda(x)=0.1290 x+$

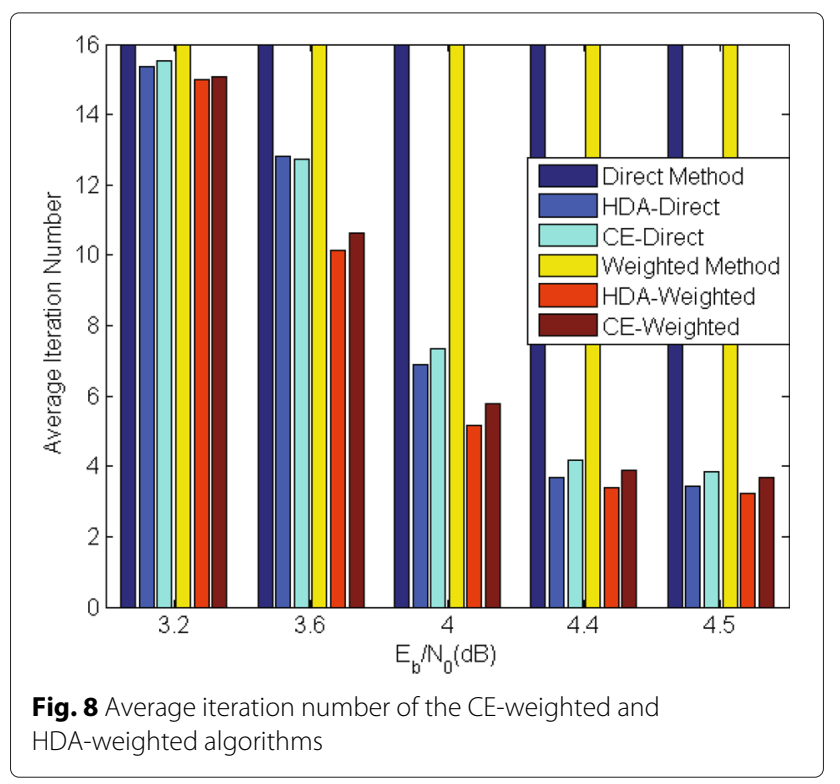




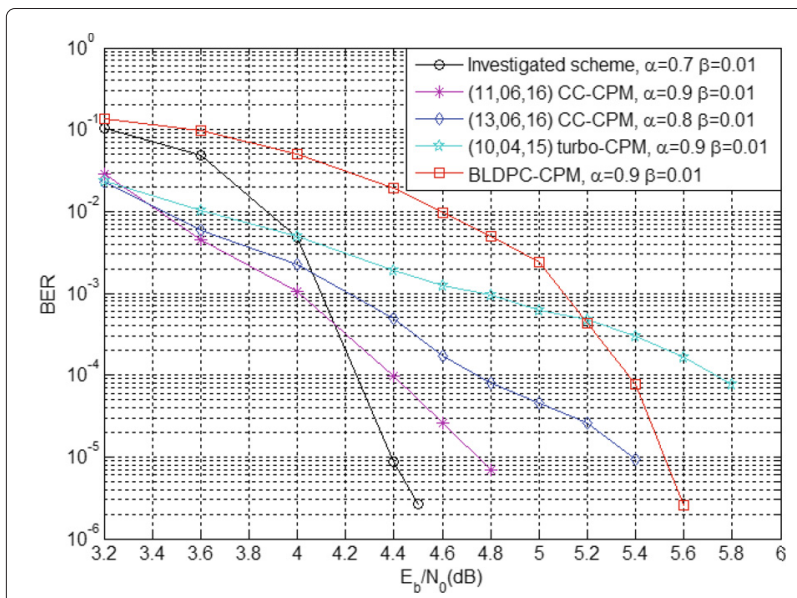

Fig. 9 BER performance of the investigated scheme and several alternative schemes

$0.4839 x^{2}+0.3871 x^{5}$ as the 8-ary LDPC code used in Fig. 6. The weighted parameters in the abovementioned schemes have been optimized using simulation experiments, as mentioned in Section 4. Figure 9 shows that CC-CPM and turbo-CPM obtain a better BER performance than the investigated scheme when $E_{b} / N_{0}$ is varied from 3.2 to $4 \mathrm{~dB}$. Meanwhile, the BER of the investigated scheme has the highest convergence speed with the increase in SNR, which is helpful in reaching a low BER order of magnitude. The investigated scheme earns gains of at least 0.3, 0.95 and $1.05 \mathrm{~dB}$ in the region of $\mathrm{BER}=10^{-5}$ against CC-CPM with $(11,06,11)$, CC-CPM with $(13,06,16)$ and BLDPCCPM schemes, respectively. In the region of $B E R=10^{-4}$, an approximate $1.5 \mathrm{~dB}$ gain is attained by the investigated scheme compared with turbo-CPM. Thus, the association between NB-LDPC codes and CPM is superior to the other candidates in the aspect of power efficiency.

\section{Conclusions}

To avoid information loss in the mutual conversion of bit and symbol probabilities when binary LDPC codes are combined with high-order CPM, the combination of NB-LDPC codes and high-order PRCPM with the same number of levels is considered a possible candidate for the uplink of satellite communications. In view of the positive feedback and relatively large decoding delay in iterative detection, we propose CE and HDA iterative stopping algorithms based on weighted extrinsic information exchange for cases with medium and high SNRs. A lot of simulation results in the slow flat Rayleigh channel demonstrate that the NB-LDPC-coded CPM scheme has better BER performance than CC-CPM, turbo-CPM, and BLDPC-CPM for the medium and high SNR scenarios. Comparison with the direct method shows that the two proposed algorithms can inhibit positive feedback and improve the power efficiency and reliability of the system we investigate. Moreover, the said algorithms also significantly reduce the average iteration number to improve the real-time performance of iterative detection.

\section{Abbreviations}

NB-LDPC: non-binary low-density parity-check; PRCPM: partial response continuous phase modulation; SNR: signal-to-noise ratio; CE: cross entropy; HDA: hard decision aided; BER: bit error rate; CPE: continuous phase encoder; MM: memoryless modulator; SCCPM: serially concatenated continuous phase modulation; MAP: maximum a posteriori; PEG: progressive edge growth; SISO: soft-input soft-output; PDF: probability density function; CSI: channel state information; BICM-ID: bit-interleaved coded modulation with iterative decoding; SCR: sign change ratio.

\section{Competing interests}

The authors declare that they have no competing interests.

\section{Acknowledgements}

This paper is supported by the National Natural Science Foundation of China (Grant No. 61403093), Science Foundation of Heilongjiang Province of China for Returned Scholars (Grant No. LC2013C22), the Assisted Project by Heilongjiang Province of China Postdoctoral Funds for Scientific Research Initiation (Grant No. LBH-Q14048) and China Fundamental Research Funds for Central Universities (Grant No. HEUCF1508).

Received: 14 July 2015 Accepted: 18 February 2016

Published online: 27 February 2016

References

1. CE Sundberg, Continuous phase modulation. IEEE Commun. Mag. 24(4), 25-38 (1986)

2. AG Amat, CA Nour, C Douillard, Serially concatenated continuous phase modulation for satellite communications. IEEE Trans. Wirel. Commun. 8(6), 3260-3269 (2009)

3. BF Beidas, S Cioni, UD Bie, A Ginesi, R lyer-Seshadri, P Kim, LN Lee, D Oh, A Noerpel, M Papaleo, A Vanelli-Coralli, Continuous phase modulation for broadband satellite communications: design and trade-offs. Int. J. Satell. Commun. Netw. 31(5), 249-262 (2013)

4. R Suffritti, F Lombardo, A Piemontese, A Vanelli-Coralli, EA Candreva, G Colavolpe, R Baroni, S Andrenacci, GE Corazza, N Alagha, in IEEE Global Commun. Conf. (GLOBECOM). Energy efficient CPM waveforms for satellite mesh networks (Anaheim, CA, 2012), pp. 3317-3321

5. P Remlein, Energy efficient continuous phase modulation signals for satellite intelligent transportation systems. IET Circ. Devices Syst. 8(5), 406-411 (2014)

6. A Emmanuele, F Zanier, G Boccolini, M Luise, Spread-spectrum continuous-phase-modulated signals for satellite navigation. IEEE Trans. Aerosp. Electron. Syst. 48(4), 3234-3249 (2012)

7. RXue, YB Sun, DF Zhao, CPM signals for satellite navigation in the $S$ and $C$ bands. Sensors. 15(6), 13184-13200 (2015)

8. BE Rimoldi, A decomposition approach to CPM. IEEE Trans. Inform. Theory. 34(2), 260-270 (1988)

9. M Baldi, F Chiaraluce, R Garello, N Maturo, I Aguilar Sanchez, S Cioni, Analysis and performance evaluation of new coding options for space telecommand links_-Part I. AWGN channels. Int. J. Satell. Commun. Netw. 33(6), 509-525 (2015)

10. RXue, DF Zhao, TL Zhu, in IEEE Int. Conf. on Wireless Commun., Networking and Mobile Computing. An improved method for the convergence of iterative detection in turbo-CPM system, (Beijing, China, 2009), pp. $445-449$

11. RXue, DF Zhao, CL Xiao, in IEEE Int. Conf. on Commun. and Technology. Power and bandwidth efficient LDPC coded CPM with iterative decoding, (Nanjing, China, 2010), pp. 1019-1022

12. RXue, CL Xiao, in IEEE Int. Conf. on Commun. and Inform. Technology. An improved iterative decoding method for LDPC coded CPM systems in Rayleigh fading channel, (Hammamet, Tunisia, 2012), pp. 341-346

13. O Abassi, L Conde-Canencia, M Mansour, E Boutillon, in IEEE Wireless Commun. and Networking Conf. Non-binary low-density parity-check coded cyclic code-shift keying, (Shanghai, China, 2013), pp. 3890-3894 
14. NB Chang, DL Romero, in IEEE Conf. Record of the Forty Sixth Asilomar Conf. on Signals, Systems and Computers (ASILOMAR). Non-binary coded modulation and iterative detection for high spectral efficiency in MIMO, (Pacific Grove, CA, 2012), pp. 458-462

15. M Baldi, F Chiaraluce, R Garello, N Maturo, I Aguilar Sanchez, S Cioni, Analysis and performance evaluation of new coding options for space telecommand links-Part II. jamming channels. Int. J. Satell. Commun. Netw. 33(6), 527-542 (2015)

16. S Nowak, G Smietanka, R Kays, in IEEE Int. Symp. on Broadband Multimedia Systems and Broadcasting (BMSB). High efficiency broadband transmission with LDPC codes over GF (2s), (Nuremberg, Germany, 2011), pp. 1-6

17. M Arabaci, IB Djordjevic, in IEEE Int. Conf. on Telecommun. in Modern Satellite Cable and Broadcasting Services (TELSIKS). Binary and nonbinary LDPC-coded modulations for generalized fading channels, (Niš, Serbia, 2011), pp. 148-151

18. R Xue, CL Xiao, in IEEE Int. Conf. on Wireless Commun., Networking and Mobile Computing. Power and bandwidth efficient q-ary LDPC coded partial response continuous phase modulation, (Shanghai, China, 2012), pp. 1-4

19. R Chaggara, ML Boucheret, C Bazile, E Bouisson, A Ducasse, JD Gayrard, in IEEE 12th European Signal Processing Conf. Continuous phase modulation for future satellite communication systems in Ka band, (Auckland, New Zealand, 2004), pp. 1083-1086

20. T Aulin, CE Sundberg, Continuous phase modulation-Part I. Full response signaling. IEEE Trans. Commun. 29(3), 196-209 (1981)

21. H Song, JR Cruz, Reduced-complexity decoding of q-ary LDPC codes for magnetic recording. IEEE Trans. Magn. 39(2), 1081-1087 (2003)

22. S Bentdetto, D Divsalar, G Montorsi, F Pollara, A soft-input soft-output APP module for iterative decoding of concatenated codes. IEEE Commun. Letters. 1(1), 22-24 (1997)

23. P Moqvist, T Aulin, Serially concatenated continuous phase modulation with iterative decoding. IEEE Trans. Commun. 49(11), 1901-1915 (2001)

24. TL Tapp, RL Mickelso, in Military Communications Conference Proceedings. Turbo detection of coded continuous-phase modulation, (Atlantic City, NJ, 1999), pp. 534-537

25. KR Narayanan, GL Stüber, Performance of trellis-coded CPM with iterative demodulation and decoding. IEEE Trans. Commun. 49(4), 676-687 (2001)

26. TRichardson, The geometry of turbo-decoding dynamics. IEEE Trans. Inform. Theory. 46(1), 9-23 (2000)

27. ZX Han, WB Bi, XZ Zhang, A method to improve the iterative detection convergence of SCCPM. J. Electron. Inf. Technol. 29(2), 274-277 (2007)

28. JH Sun, ZY Li, Stopping algorithm for iterative decoding based on the average extrinsic information exchange for SCCPM. J. Xidian University (Natural Science). 35(4), 716-720 (2008)

29. S Benedetto, D Divsalar, G Montorsi, F Pollara, A soft-input soft-output maximum a posteriori (MAP) module to decode parallel and serial concatenated codes. TDA Prog. Rep. 42(127), 1-20 (1996)

30. J Hagenauer, E Offer, L Papke, Iterative decoding of binary block and convolutional codes. IEEE Trans. Inform. Theory. 42(2), 429-445 (1996)

31. RY Shao, S Lin, M Fossorier, Two simple stopping criteria for turbo decoding. IEEE Trans. Commun. 47(8), 1117-1120 (1999)

32. S Zhang, JP Li, CS Cai, in IEEE Int. Conf. on Wireless Commun. \& Signal Processing (WCSP). A variable iterative decoding scheme for BICM-ID based on cross-entropy, (Nanjing, China, 2009), pp. 1-4

33. YF Wu, BD Woerner, WJ Ebel, A simple stopping criterion for turbo decoding. IEEE Commun. Letters. 4(8), 258-260 (2000)

34. ZX Han, WB Bi, XZ Zhang, in IET Int. Conf. on Wireless, Mobile and Multimedia Networks. An improved iterative detection method for serially concatenated CPM, (Hangzhou, China, 2006), pp. 1-4

35. R Xue, CL Xiao, in Int. Conf. on Wireless Commun., Networking and Mobile Computing (WiCOM). A joint coded modulation scheme and its iterative receiving for deep-space communications, (Shanghai, China, 2012), pp. $1-4$

\section{Submit your manuscript to a SpringerOpen ${ }^{\circ}$ journal and benefit from:}

- Convenient online submission

- Rigorous peer review

- Immediate publication on acceptance

- Open access: articles freely available online

- High visibility within the field

- Retaining the copyright to your article

Submit your next manuscript at $>$ springeropen.com 